\title{
Canonical Statistical Model for Maximum Expected Immission of Wire Conductor in an Aperture Enclosure
}

\author{
Paul G. Bremner \\ Robust Physics \\ Del Mar, CA 92014 USA \\ pbremner@robustphysics.com
}

\author{
Gabriel Vazquez, Daniel J. Christiano, Dawn H.Trout \\ NASA Kennedy Space Center \\ Cape Canaveral, FL, USA \\ gabriel.vazquez@nasa.gov
}

\begin{abstract}
Prediction of the maximum expected electromagnetic pick-up of conductors inside a realistic shielding enclosure is an important canonical problem for system-level EMC design of space craft, launch vehicles, aircraft and automobiles. This paper introduces a simple statistical power balance model for prediction of the maximum expected current in a wire conductor inside an aperture enclosure. It calculates both the statistical mean and variance of the immission from the physical design parameters of the problem. Familiar probability density functions can then be used to predict the maximum expected immission for deign purposes. The statistical power balance model requires minimal EMC design information and solves orders of magnitude faster than existing numerical models, making it ultimately viable for scaled-up, full system-level modeling. Both experimental test results and full wave simulation results are used to validate the foundational model.
\end{abstract}

Keywords - Statistical electromagnetics, Reverberant field, Immission, Emission, Maximum expected field strength, Stochastic EMC

\section{INTRODUCTION}

Statistical model-based EMC design is evolving for complex electronic networks installed in enclosed spaces typical of spacecraft, aircraft and automobiles. The process starts with prediction of the electromagnetic field strength in each approximately reverberant EM field domain in the vehicle. At high frequencies and in electrically large domains, both temporal and spatial field response is highly sensitive to small geometric details which are uncertain within normal manufacturing tolerances; and which are not repeatable experimentally. Given this uncertainty it is not practical to model design details explicitly. For design purposes, it is only meaningful to predict the statistical time average and space-average mean electric field, and the maximum expected field strength.

Bremner [3] has shown how a modal expansion basis for each reverberant EM field can be used to predict the first two statistical moments of the response - mean and variance - from the physical EM parameters of each subsystem. With selection of a suitable probability density function [1][2][4], it is then possible to predict both mean and maximum expected response in each domain. Bremner showed that a Log Normal distribution for the total energy of a reverberant enclosure provides a good estimate of maximum predicted electric field response in three different size reverberant volumes. In the EMC design process, the predicted maximum expected electric field is normally used to define the radiation immunity test specification for all electronic units and network systems operating in that domain
In the common event that certain critical electronic units or subsystems cannot be easily "test qualified" or re-designed to operate in the maximum expected EMC environment, it often becomes necessary to design EM field attenuation measures, typically in the form of shielding enclosures, avionics boxes, etc. The EMC design problem is to define the amount of EMC attenuation required for the critical electronic unit(s) - typically quantified as the shielding effectiveness. To do this, electronic system designers need a simple means to estimate the EM field induced currents (both differential and common mode) in power lines and signal (transmission) lines for both external cables and traces on printed circuit boards. At high frequencies and for electrically large shielding enclosures, the spatiotemporal EM field response will be sensitive to uncertain geometric details. In the presence of this inherent uncertainty, the only statistically meaningful model is one that seeks to predict only the mean and maximum expected response in wire conductors.

This paper introduces a simple statistical power balance model for prediction of the maximum expected current in a wire conductor inside an aperture enclosure. It calculates both the statistical mean and variance of the immission entirely parametrically - ie. from only the physical design parameters of the problem. Familiar probability density functions are then used to predict the maximum expected immission for deign purposes. The statistical power balance model requires minimal EMC design information and solves orders of magnitude faster than existing numerical models, making it ultimately viable - in principle - for scaled-up, full system-level modeling

\section{FOUNDATIONS OF STATISTICAL EMC MODELING}

Hill [1] has laid the foundations for statistical power balance modeling of EM fields in reverberant, multi-modal domains. While full wave simulation tools are an alternative model-based design approach, Trout [2] and others have shown that deterministic models do not reliably estimate the predicted EM field at a point, without statistical reduction to account for uncertainties in the EM source field and uncertainty in EM parameters of the reverberant domain.

Junqua, et al [3] has shown how Hill's statistical power balance approach could be applied to a quite general network of reverberant (multi-modal) electromagnetic subsystems. Junqua's approach has been applied to modeling the EM environments in connected cavities, typical of a space launch vehicle payload fairing [4]. Encouraging results were obtained for the statistical mean (space-time average) electric field in each subsystem. However for space vehicle EMC design purposes, it is important to also be able to predict the maximum expected EM field strength.

Kovalevsky et al [6] have recently shown that the statistical 
energy analysis model can predict the mean and variance of EM response for an aperture cavity in a reverberation chamber. The authors suggest that the predicted statistical moments can be used with a "double Raleigh distribution" reported by others to predict the maximum expected field strength in the aperture cavity. However, no results are presented to show validation of this aspect of the model.

This paper extends the foregoing research work to validate statistical power balance modeling approach for prediction of the statistical mean and maximum expected current induced in both the shielding enclosure and in a wire conductor located within an aperture cavity, as shown in Figure 2 below. The statistical model results are compared with S-parameter measurements and full wave EM simulation results for configurations similar to those studied by Tait [5] and by Rajamani et al [7].

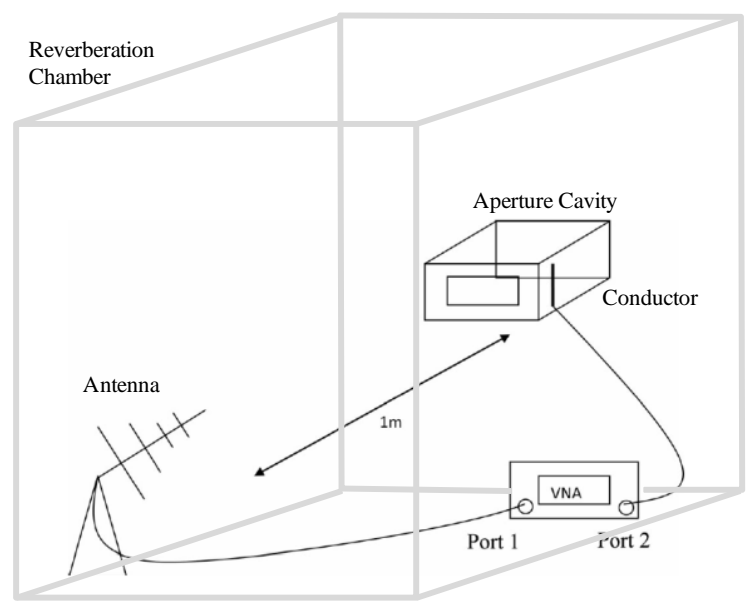

Figure 1 Test configuration for wire conductor pick-up in an aperture cavity which is described by Mean and Max response predictions of s Statistical EM model.

\section{STATISTICAL MODEL}

As for reverberation chambers we can adequately quantify the electric field by one of three parameters - the total electromagnetic energy $U$ (or energy density $W=U / V$ ) of the reverberant field; or the total magnitude of the electric or magnetic field strength $E_{T}$, $H_{T}$; or the magnitude of a vectorial component of the electric or magnetic field; eg. $E_{x}, E_{y}$ and $E_{z}$. The total electromagnetic energy [10] is

$$
U=\frac{1}{2} \int_{V}\left(\varepsilon\left|E_{T}\right|^{2}+\mu\left|H_{T}\right|^{2}\right) d V \equiv \int_{V} \varepsilon\left|E_{T}\right|^{2} d V
$$

For a statistically uniform electric field in a well-stirred reverberation chamber, it has been shown [10] that the power balance principle applies

$$
P_{\text {in }}=P_{\text {diss }}=\omega \eta U=\omega U / Q
$$

That is, the power input from an external source $P_{i n}$ must be balanced by the total power dissipated by all losses, where $Q$ is the cavity $Q$ factor ( $\eta$ is the corresponding damping loss factor $\eta=1 / Q)$

\section{A. Subsystem Energy Definitions.}

The total energy of the reverberation chamber is

$$
U_{R}(\omega)=\varepsilon V_{R}\left|E_{T, R}\right|^{2}
$$

where $\varepsilon$ is the emissivity of air, $V_{R}$ is the volume of the chamber and $\left|E_{T, R}\right|^{2}$ is the space-time average of the mean-squared total electric field vector in the chamber.

Likewise, the total energy of the aperture cavity is

$$
U_{C}(\omega)=\varepsilon V_{C}\left|E_{T, C}\right|^{2}
$$

The total energy of the wire conductor is

$$
U_{W}(\omega)=\mu A_{w} L_{w}\left|H_{T, W}\right|^{2}
$$

where $\mu$ is the permittivity of air surrounding the wire, $A_{w}, L_{w}$ are the cross section area and length of the wire and $\left|H_{T, W}\right|^{2}$ is the mean-squared total magnetic field vector in the chamber.

\section{B. System Power Balance equations.}

For multiply-connected reverberant subsystems, there is a power balance equation for each that will include energy transmitted to connected systems, a shown in Figure 2.

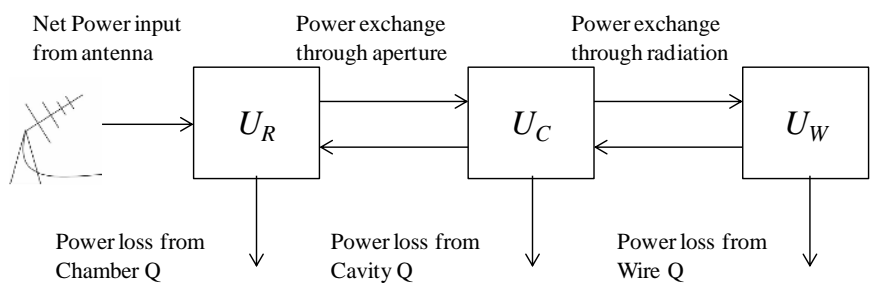

Figure 2 Power balance schematic for the canonical model

In the field of vibro-acoustics, Lyon [11] has shown that the net power flow $\left\langle P_{i j}\right\rangle$ between any two reverberant energy subsystems (i.j) - averaged over an ensemble of uncertain parameters - is proportional to the difference in their modal energy levels

$$
\left\langle P_{i j}\right\rangle=\omega n_{i} \eta_{i j}\left\{\frac{\left\langle U_{i}\right\rangle}{n_{i}}-\frac{\left\langle U_{j}\right\rangle}{n_{j}}\right\}
$$

where $n_{i}(\omega)=\Delta N_{i} / \Delta \omega$ is the modal density and $\eta_{i j}(\omega)$ is a frequency-dependent coupling loss factor. Writing the power balance equations for each of the three subsystems in the canonical model yields a power balance matrix which can be solve for the average reverberant energy levels $\left\langle U_{R}\right\rangle,\left\langle U_{C}\right\rangle,\left\langle U_{W}\right\rangle$

$$
\left\{\begin{array}{c}
P_{R}^{i n} \\
0 \\
0
\end{array}\right\}=\omega\left[\begin{array}{ccc}
\eta_{R}+\eta_{R C} & -\eta_{C R} & 0 \\
-\eta_{R C} & \eta_{C}+\eta_{C R}+\eta_{C W} & -\eta_{W C} \\
0 & -\eta_{C W} & \eta_{W}+\eta_{W C}
\end{array}\right]\left\{\begin{array}{l}
U_{R} \\
U_{C} \\
U_{W}
\end{array}\right\}
$$




\section{Loss Factor Notation.}

Previous work on statistical power balance modeling used Q as the quantity describing energy dissipation or damping and effective cross section $\left\langle\sigma_{i j}\right\rangle$ in units of area, to quantify strength of coupling energy transmission. In this model, we prefer to use dimensionless loss factor notation for each. The damping or dissipation loss factor $\eta_{j}$ is simply the inverse of the $Q_{j}$ factor. Likewise, there are simple linear conversions between coupling loss factors $\eta_{i j}$ and coupling effective section (area). The loss factor notation has some diagnostic advantages, as will be seen from the analysis presented in this paper.

Coupling loss factors defined in this way satisfy the reciprocity relationship

$$
n_{i} \eta_{i j}=n_{j} \eta_{j i}
$$

\section{Parametric Variance Model}

For reverberant wave fields in perfectly square or rectangular domains, Lyon [11] provides a non-parametric variance formulation based on a Poisson distribution of natural frequency spacings. For more realistic structures with quite general and irregular boundaries, Weaver [12] has shown that the natural frequency spacings are more correctly described by the Gaussian Orthogonal Ensemble (GOE) from random matrix theory. Weaver provides a non-parametric variance formulation for the energy density of a reverberant wavefield excited by one or more point excitations. The relative variance $r^{2}[U]=\operatorname{Var}[U] /\langle U\rangle^{2}$ takes the form

$$
r^{2}[U]=\frac{1}{L N}+\frac{1}{\pi m}\left\{\left(\frac{K}{N}+1-\frac{1}{N}\right)\left(\frac{K}{L}+1-\frac{1}{L}\right)-\frac{2}{L N}-1\right\}
$$

where $\mathrm{L}$ and $\mathrm{N}$ are respectively the number of receiver and source positions used to calculate cavity energy. The "modal overlap" $m$ is the principal term describing the uncertainty in resonance frequency. Modal overlap is a function of the frequency, the mean damping loss factor $\eta_{j}$, and the modal density $\left(n_{j}\right)$ of the reverberant enclosure [11][12][13]

$$
m_{j}(\omega)=\omega n_{j}(\omega) \eta_{j}(\omega), \text { where } n_{j}=V_{j} \omega^{3} / \pi^{2} c^{3}
$$

The parameter $K$ is a measure of the spatial variance of the cavity mode shapes $\psi_{r}(x)$ which asymptotes to a value defined by wavefield dimensionality [11]

$$
K=E\left[\psi_{r}^{4}\right] / E\left[\psi_{r}^{2}\right]^{2} \cong 3
$$

For the case where the applied excitation voltage is measured or controlled, Langley and Brown [14] have shown that:

$$
\operatorname{Var}\left[P_{i n, \Delta \omega}\right]=\left\langle P_{i n, \Delta \omega}\right\rangle r^{2}(\alpha, m, B)
$$

where the $r^{2}$ term represents the relative variance of the modal radiation resistance as a function of the variance in coupling between excitation spatial correlation and mode shape $(\alpha)$, a frequency averaging bandwidth parameter $(B)$, and the modal overlap $(m)$. The $\alpha$ term plays the same role as the $K$ term in (9), for the case of single point excitation.
The frequency averaging bandwidth parameter may be determined as a function of the frequency bandwidth $\Delta \omega$ and the $Q$ factor using $B=\Delta \omega / \eta \omega$. The excitation relative variance is $\alpha=E\left[\left(j_{r}^{2}\right)^{2}\right] / E\left[\left(j_{r}^{2}\right)\right]^{2}$ where $j_{r}^{2}$ is the modal joint acceptance, a spatial double integral of the excitation spatial correlation and the $r t h$ mode shape of the reverberant cavity. Langley and Brown have shown that the variance of this band-averaged complex modal integral converges smoothly to asymptotic values in the range $\alpha=1-3$, which can be determined from the number of spatial degrees of freedom associated with the excitation and from the known dimensionality of the reverberant wavefield.

For a frequency band integrated estimate of the input power, the relative variance term may be determined from Langley and Brown [14] using:

$$
\begin{aligned}
r^{2}(\alpha, m, B)= & \frac{\alpha-1}{\pi m}\left(\frac{1}{B^{2}}\right)\left\{2 B\left[\frac{\pi}{2}-\tan ^{-1}\left(\frac{1}{B}\right)\right]-\ln \left(1+B^{2}\right)\right\} \\
& +\frac{1}{(\pi m)^{2}}\left(\frac{1}{B^{2}}\right) \ln \left(1+B^{2}\right)
\end{aligned}
$$

\section{TEST CONFIGURATION}

The canonical statistical model is compared with measurements taken in a reverberation chamber at NASA Kennedy Space Center, shown in Figure 3 below.

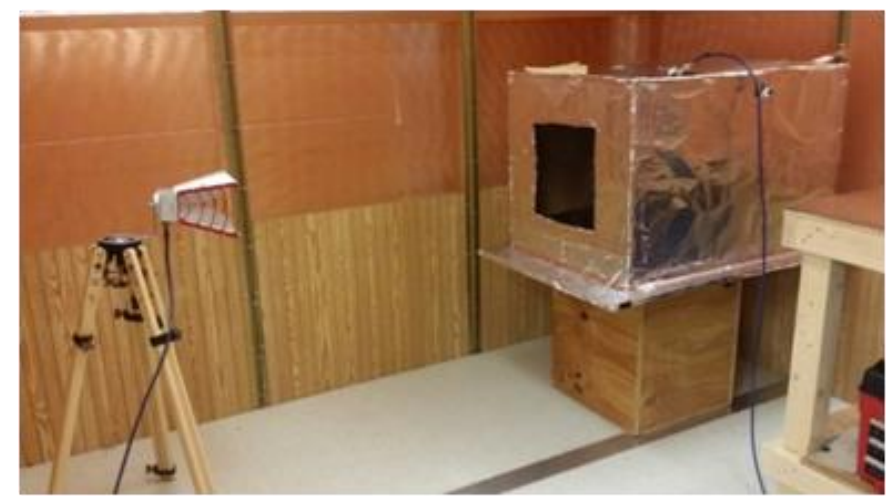

Figure 3 Canonical problem test configuration in NASA KSC reverberation chamber.

The reverberation chamber dimensions are height 2.31 meters, width 3 meters and depth (length) 5.035 meters. The walls are lined with copper mesh. The aperture cavity is cardboard box covered inside and outside with aluminum foil. The box dimensions are height 0.665 meters, width 0.67 meters and depth (length) 0.98 meters. Apertures of different sizes were tested, ranging from a small circular aperture of $0.20 \mathrm{~m}$ radius to a large square aperture $0.35 \mathrm{~m} \times 0.35 \mathrm{~m}$.

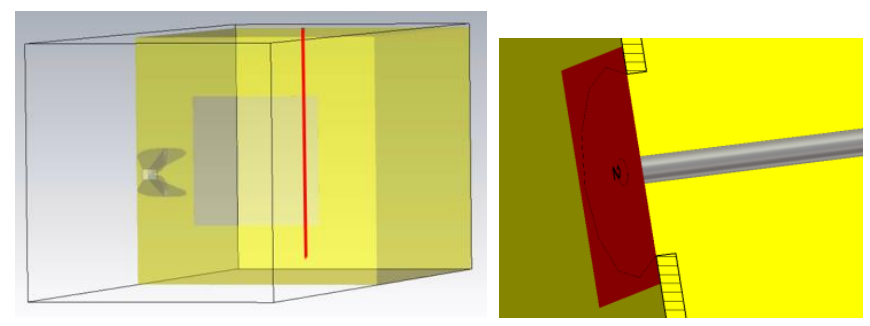


Figure 4 Wire conductor as mounted in one of nine positions in top surface of aperture cavity (left) and $50 \mathrm{Ohm} \mathrm{SMA} \mathrm{connection} \mathrm{to} \mathrm{surface} \mathrm{(right).}$

The wire conductor is a $1 \mathrm{~mm}$ radius copper wire of length 60 $\mathrm{mm}$. The wire is connected to the top aluminum surface of the aperture cavity via a $50 \mathrm{Ohm}$ SMA connector. The aluminum conducting surface of the cavity forms an effective ground plane such that the electromagnetic reception of the wire conductor is somewhat similar to that of a traveling wave antenna.

A statistical ensemble of results for the electromagnetic field strength in the RC and the aperture cavity is obtained by using a mode-strirrer in the reverberation chamber, as shown in Figure 5 (left). A statistical ensemble of results for the electromagnetic field strength in the aperture cavity and the wire conductor is obtained by measuring the wire S-parameter $|\mathrm{S} 21|$ at up to nine different positions in the aperture cavity, as shown in Figure 5 (right) .
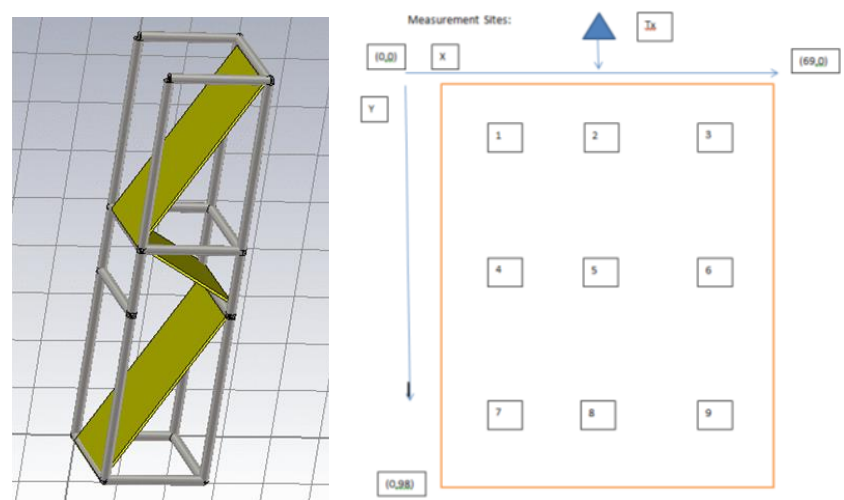

Figure 5 Mode strirrer used in reverberation chamber (left) and array of nine locations of wire conductor in aperture cavity (right)

\section{A. $\quad$ Estimated $Q$ factors}

The estimated $\mathrm{Q}$ factors for each of the three subsystems is shown Figure 6 below.

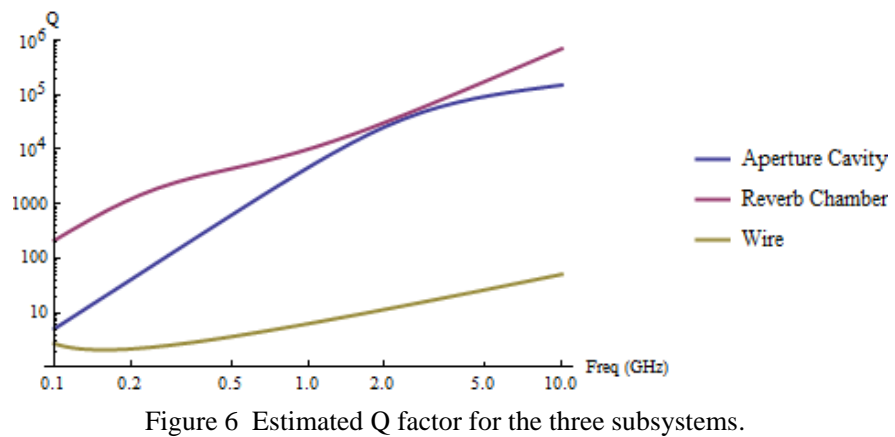

\section{B. Modal Overlap}

The modal overlap $m_{\mathrm{j}}$ is calculated from the estimated $\mathrm{Q}$ factor and the corresponding modal density for each reverberant subsystems. Results are shown in Figure 6 below.

This is a significant result. A modal overlap equal or greater than approximately unity ensures that the parametric variance models are valid. The modal overlap of the RC asymptotes to unity because losses from the source antenna control $\mathrm{Q}$ at low frequencies. Likewise, losses due to radiation to the wire conductor controls the cavity $\mathrm{Q}$ at low frequencies. The guided electromagnetic field on the wire conductor has a low Q since it is typically connected to an approximately matched, load impedance.

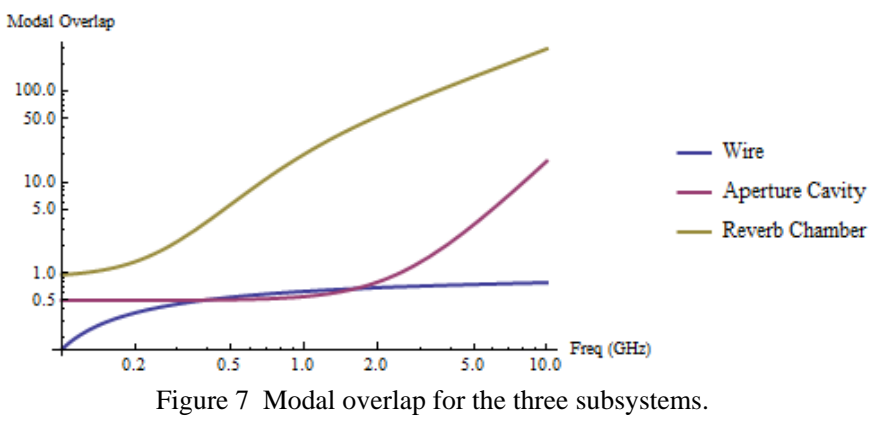

\section{C. $\quad$ Energy in Aperture Cavity}

The power balance model was used to predict the electromagnetic field strength in the cavity, with a $20 \mathrm{~mm}$ radius circular aperture as reported by Kovalevsky [4] The result in Figure 8 demonstrates that model predictions for the mean total energy are in good agreement with test results.
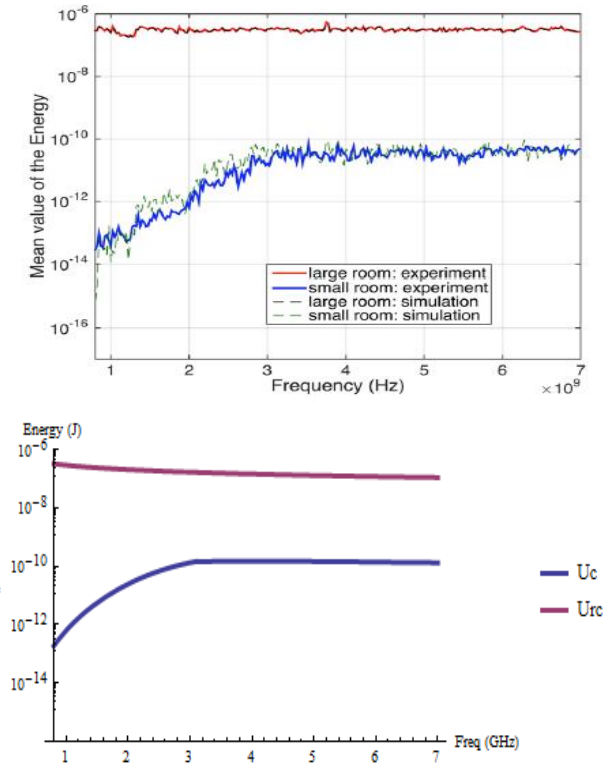

Figure 8 Electromagnetic energy levels in Reverb Chamber (red) and Aperture Cavity (blue); measured (top) and predicted (bottom)

\section{Power Balance Design Diagnostics}

A strong attribute of the statistical power balance model is easy access to useful design diagnostics. The relative power losses from the RC in Figure 9 below, shows that electric field strength is controlled by the wall losses; little is lost through the cavity aperture.

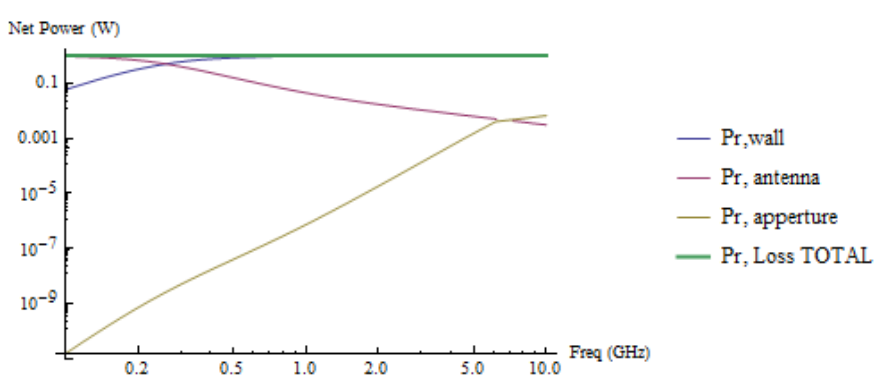


Figure 9 Relative EM power losses which control the electric field strength in the Reverberation Chamber, for unit power input from source antenna

\section{E. EM Pick-up (Immission) in Wire Conductor}

The power balance model was used to predict the electromagnetic field strength in the cavity, with a $20 \mathrm{~mm}$ radius circular aperture as reported by Kovalevsky [4] The result shown in Figure 10 below.

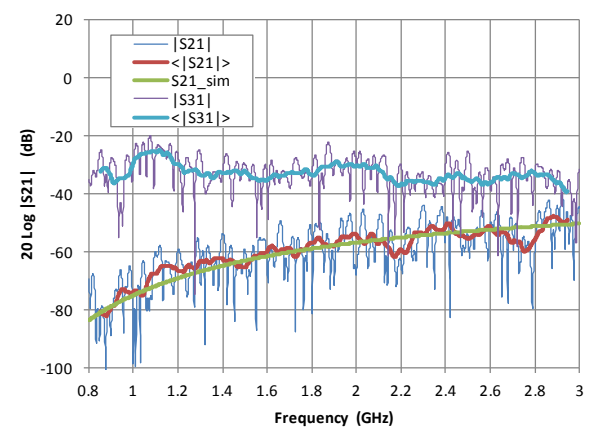

Figure $10 \mathrm{~S}$-parameters from source antenna to wire conductor in aperture cavity (S21) and from source antenna to reference antenna in RC (S31)

\section{F. $\quad$ Evaluation of Reberberant Field Assumption}

The assumption of an approximately diffuse reverberant EM field in the aperture cavity was investigated by evaluation S21 at nine different wire conductor locations, with the largest $0.35 \mathrm{~m} \times 0.35 \mathrm{~m}$ square aperture. The result in Figure 11 shows that the spatial variance in the wire conductor immission is smaller than the frequency-tofrequency modal variance, justifying the diffuse field assumption.

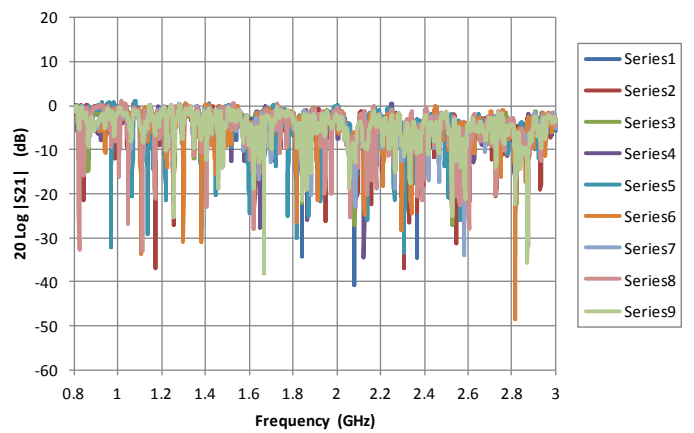

Figure 11 S21 results superimposed for nine different wire conductor locations in the aperture cavity

\section{G. $\quad$ Variance and Maximum Expected Response}

The statistical variance of this ensemble was found to be predictable from the reverberant energy variance. The Langley and Brown energy variance formulation correctly predicted the variance at low frequencies, but collapsed quickly to zero variance as the modal overlap increased at higher frequencies.

The Weaver energy variance formulation was found to provide a better estimate of $\operatorname{Var}[U]$ over the whole measured frequency range, because equation (9) retains a $1 / L N$ term that captures the residual variance of a finite sample of data used to define the energy $U$. For a single point exciter $L=1$ and for thirty electric field measurement probes, the parameter $L N=30$.

The graphs of $\log \left(A v g\left[E_{r}\right]\right)$ in $\mathrm{dB}$ - which is proportional to $\log [U]$ - exhibit an approximately normal distribution about the mean, consistent with the common assumption that the energy $U$ is log-normally distributed.

To predict the maximum expected energy from the log normal distribution, it was first necessary to transform the predicted mean $\langle U\rangle$ and the predicted variance $\operatorname{Var}[U]^{\text {to }}$ the log domain, using [15]

$$
10\langle\log (U)\rangle=10 \log \langle U\rangle-5 \log \left(1-r^{2}[U]\right)
$$

and

$$
\operatorname{Var}[\log (U)]=43 \log \left(1+r^{2}[U]\right)
$$

For this distribution, the maximum expected $\log [U]$ with $97.5 \%$ probability is

$$
P 97.5[\log [U]]=\langle\log [U]\rangle+1.96\{\operatorname{Var}[\log [U]]\}^{0.5}
$$

and the minimum expected $\log [U]$ with $2.5 \%$ probability is

$$
P 2.5[\log [U]]=\langle\log [U]\rangle-1.96\{\operatorname{Var}[\log [U]]\}^{0.5}
$$

The mean, P97.5 maximum expected and P2.5 minimum expected predictions for the corresponding $\log \left(\operatorname{Avg}\left[E_{r}\right]\right)$ are a good fit to the observed EM field statistics, as shown (overlaid) in Figure 12.

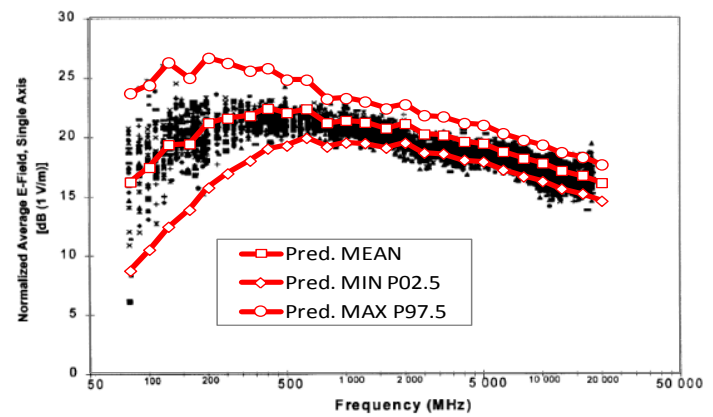

Figure 12 Predicted mean, maximum expected [P99.5] and minimum expected [P2.5] for $A v g\left[E_{r}\right]$ ensemble, in the RC

\section{CONCLUSION}

A simple statistical power balance model has been introduced for predicting mean and maximum expected voltage induced in a wire conductor shielded from a strong electromagnetic field by an enclosure. Preliminary comparisons with experiments and full wave numerical modeling results are promising. The model can predict the shielding effectiveness of the imperfect (aperture) enclosure and can predict the maximum expected current induced in a wire conductor in the shielding enclosure, even at low frequencies where modal overlap is less than unity.

While this is a very simple configuration, it is important to note that the statistical power balance model requires minimal EMC design information and solves orders of magnitude faster than existing numerical models. As such, this canonical model demonstrates that statistical EMC modeling is potentially viable for scaled-up, full system-level modeling of complex electronic networks in spacecraft, aircraft and automobile.

\section{ACKNOWLEDGEMENT}

The authors gratefully acknowledge funding for this work by NASA Kennedy Space Center under Special Projects contracts, including Contract No. NNK15LA78P. 


\section{REFERENCES}

[1] I. Junqua, J.P. Parmentier, F. Isaac "A Network Formulation of the Power Balance Method for High-Frequency Coupling" Electromagnetics, vol. 25, pp 603-622, 2007.

[2] A. Schaffar and P. N. Gineste, "Application of the power balance methods to E- field calculation in the ARIANE 5 launcher payloads cavities," Presented at International Symposium on EMC, Long Beach, 2011

[3] P.G. Bremner, "Modal Expansion Basis for Statistical Estimation of Maximum Expected Field Strength in Enclosures", Proc. IEEE EMC Symposium, Santa Clara, March 2015

[4] L. Kovalevsky, R.S. Langley, P. Besnier and J. Sol, "Experimental validation of the Statistical Energy Analysis for coupled reverberant rooms", Proc. IEEE Intnl. Symp. EMC, Dresden, August 2015

[5] G.B Tait, C. Hager, M.B. Slocum and M.O. Hatfiled, "On Measuring Shielding Effectiveness of Sparsely Moded Enclosures in a Reverberation Chamber", IEEE Trans. on EMC, Volume:55, Issue: 2, October 2012

[6] G.B. Tait, R.E. Richardson, M.B. Slocum, M.O. Hatfield and M.J. Rodriguez, "Reverberant Microwave Propagation in Coupled Complex Cavities" IEEE Trans. on EMC, Vol. 53, No. 1, February 2011
[7] V. Rajamani, J.C. West and C.F. Bunting, "Measurement and Simulation of the Induced Current on a Wire Using S-Parameter Method", Trans. IEEE on EMC, VOL. 56, NO. 2, APRIL 2014

[8] Coffee E.L., Tucker A.E. and Johnson, R.M. "Using Statistical Electromagnetics for EMC/HERO Analysis", Proc. US Air Force 15th Aircraft / Stores Compatibility Symposium, 2010.

[9] D.H. Trout, "Electromagnetic Environment In Payload Fairing Cavities," Dissertation, University of Central Florida, 2012.

[10] D. A. Hill, "Electromagnetic Fields in Cavities. Deterministic and Statistical Theories" John Wiley \& Sons, Hoboken, New Jersey 2009

[11] R.H. Lyon, Statistical analysis of power injection and response in structures and rooms", Jnl. Acoust. Soc. Amer. (1969) 45(3), 545-565

[12] R. Weaver, "On the ensemble variance of reverberation room transmission functions, the effect of spectral rigidity", Jnl. Sound \& Vibration (1989) 130(3), 487-491

[13] R.S. Langley and Brown, "The ensemble statistics of the energy of a random system subjected to harmonic excitation", Jnl. Sound \& Vibration (2004) 275, 823-846

[14] R.S. Langley. and A.W.M. Brown, "The ensemble statistics of a bandaveraged energy of a random system", Jnl. Sound and Vibration, 275, pp 847-857, 2004

[15] R.H. Lyon, R.G. Dejong, "Theory and Application of Statistical Energy Analysis", 2nd Edition, Butterworth-Heinemann, London, 1995 